\title{
Genetically targeting new neurons in the adult hippocampus
}

\author{
Zhengang Yang ${ }^{1}$, Guo-Li Ming ${ }^{2,3,4}$, Hongjun Song $2,3,4$ \\ ${ }^{I}$ Institutes of Brain Science and State Key Laboratory of Medical Neurobiology, Fudan University, Shanghai 200032, China; ${ }^{2} I n s t i-$ \\ tute for Cell Engineering, ${ }^{3}$ Department of Neurology, ${ }^{4}$ Department of Neuroscience, Johns Hopkins University School of Medicine, \\ Baltimore, MD 21205, USA
}

Cell Research (2011) 21: 220-222. doi:10.1038/cr.2010.148; published online 2 November 2010

Neurogenesis, the birth of new neurons from neural stem cells, is known to occur throughout life in two specific regions of the adult mammalian brain, the subgranular zone/hippocampal dentate gyrus and the subventricular zone/olfactory bulb [1-3]. Adult neurogenesis is a multi-step process (Figure 1), which includes proliferation and fate specification of adult neural stem cells, morphogenesis, migration, axonal and dendritic development, survival, and synaptic integration by newborn neurons [4]. Understanding the basic process and molecular mechanisms of adult neurogenesis not only enriches our general knowledge about this unique type of structural and functional plasticity in the mature brain, but also has significant implications for cell replacement therapy after injury or degenerative neurological disorders. The rapid progress made in understanding adult neurogenesis has been driven by new analytical tools introduced to the field, including labeling of proliferating progenitors using nucleotide analogs, such as Bromodeoxyuridine (BrdU), or 5-ethynyl-2'-deoxyuridine (EdU), and oncoretroviruses for quantitative

Correspondence: Zhengang Yanga ${ }^{a}$ Hongjun

Song ${ }^{\mathrm{b}}$

aE-mail: yangz@fudan.edu.cn

bE-mail: shongju1@jhmi.edu analysis of cell proliferation, fate mapping and neuronal development [5]. In this issue of Cell Research, Xiong and colleagues bring a significant new addition to our tool box with a genetic approach, based on inducible Cre/loxP recombinase, for pulse labeling, longterm tracing and genetic manipulation of newborn neurons in the dentate gyrus of adult mice in vivo [6].

A major roadblock in our ability to investigate adult neurogenesis in vivo has been the limitation of available methods for lineage tracing and genetic manipulation of specific neural progenitor subtypes in the intact brain [5]. As a result, many questions remain to be answered in the field. For example, what are the contributions of newborn neurons to specific brain functions and behaviors? What are the molecular and cellular mechanisms regulating the development of new neurons? Nucleotide analogs BrdU or EdU, which are permanently incorporated during the $\mathrm{S}$ phase of cell cycle, are widely used as a means for birth-dating and quantification of proliferation and fate choice of neural progenitors at the population level [5]. This methodology, however, does not allow for genetic manipulation or analysis of morphology and physiological properties of labeled neural progeny. Although the retroviral approach overcomes many of the shortcomings of the
BrdU/EdU method through expression of fluorescent markers and transgenes or short hairpin RNAs [7], it has its own limitations. Only a small number of cells can be manipulated and the approach is invasive. Recently, inducible Cre/loxP recombinase systems in transgenic mice based on prompters for human glial fibrillary acidic protein (hGFAP), nestin and L-glutamate/L-aspartate transporter (GLAST) have been developed for labeling and manipulation of neural progenitors in the adult brain. These transgenic mice are amendable for activating or inactivating genes of interest in adult neural progenitors at the population level, and/or mapping their fate when a reporter gene is employed [8]. All these mice were designed to target the adult neural stem cell pool, which continuously give rises to newborn neurons and potential glia with mixed birthdates. It will be very useful to label and manipulate only newly born neurons with a defined birthdate, but not stem/progenitor cells or glia progeny in the adult brain.

Xiong and colleagues developed a transgenic mouse line that expresses the tamoxifen-inducible recombinant $\mathrm{CreER}^{\mathrm{T} 2}$ under the control of the BAC-based promoter of doublecortin (DCX; referred to as DCX-CreER mice thereafter) [6]. DCX, a microtubuleassociated protein important for neu- 


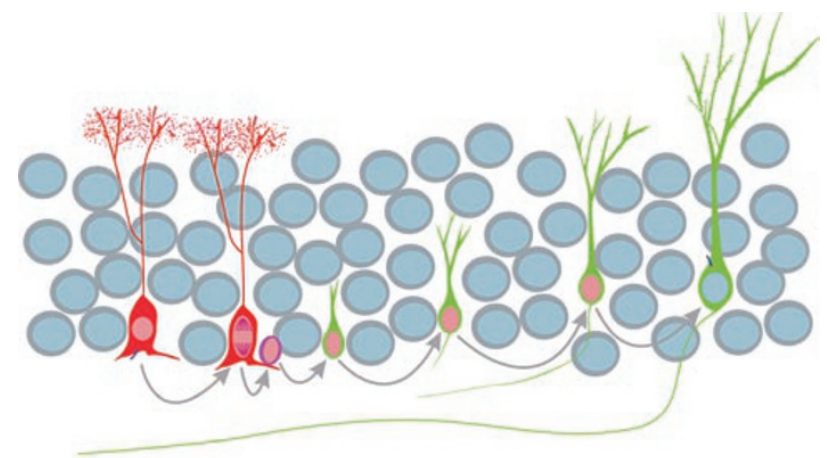

Figure 1 Adult neurogenesis is a multi-step process. In the hippocampal dentate gyrus, radial glia-like cells (red) in the subgranular zone serve as the neural stem cells. These neural stem cells give rise to transit amplifying cells (magenta), which in turn generate immature neurons (green) that express DCX, PSANCAM and Tuj1. These immature neurons (newly born neurons) then express a mature neuronal marker, NeuN, and progressively acquire electrophysiological characteristics of mature dentate granule cells (green with a grey nucleus) of the dentate gyrus. The axons of newly born mature granule cells successfully establish synapses with hilar interneurons and CA3 pyramidal neurons of the hippocampal formation. DCX-CreER mice generated by Cheng $X$ et al. [6] can be used for specifically labeling and manipulating newly born neurons (green) in the hippocampus of the adult mouse brain.

ronal migration, is specifically and transiently expressed in proliferating neuroblasts and newborn immature neurons in both developing and adult brain [9]. DCX has been widely used as an immunohisotological marker for newborn neurons in the adult neurogenic regions [9]; transgenic mice expressing fluorescent proteins under the control of BAC-based or a short promoter of DCX have been developed to transiently mark newborn neurons in the adult brain [10]. In the DCX-CreER mice, the recombination is temporally controlled by the tamoxifen administration and spatially restricted to immature neurons. Importantly, the labeling is permanent once the recombination occurs. By crossing the DCX-CreER mice with reporter mice for inducible expression of yellow fluorescent protein, the authors provided evidence that a majority of labeled neurons are born within a 10 day window before induction [6]. Thus, a cohort of immature neurons with relatively defined birthdates can be selectively pulse-labeled for long-term tracing and for analysis of their morphological and electrophysiological properties in the adult dentate gyrus [6]. Intriguingly, DCX expressing neuroblasts in the subventricular zone, the largest germinal region in the adult brain which gives rise to newborn interneurons in the olfactory bulb, were not labeled after tamoxifen induction in these mice. We have independently generated multiple lines of BAC based DCX-CreER ${ }^{\mathrm{T} 2}$ mice [11]. Interestingly, only the DCX expressing newborn neurons in the adult subventricular zone are targeted in our mice, not in the adult dentate gyrus. Since the $\mathrm{CreER}^{\mathrm{T} 2}$ was not knocked into the endogenous DCX locus in mice generated from either laboratory, the differences may reflect regional insertion effects of BAC transgenes. Nevertheless, these two complementary lines of transgenic mice provide exciting genetic tools to independently target two specific populations of newborn neurons in the adult brain.

One immediate application of the DCX-CreER mice is to mark newborn neurons for analysis. Xiong and colleagues confirmed early findings that newly generated dentate granule cells exhibit high-input resistance and high excitability [12] and that a majority of these new neurons eventually migrated only to the inner third of the granule cell layer in the adult mouse hippocampus $[13,14]$. In these mice no GFAP expressing glia cells were labeled, yet a few GAD67 expressing neurons were marked [6]. Whether this limited number of $\mathrm{GAD} 67^{+}$neurons represents a transient phase of GAD67 expression in newborn dentate granule neurons, or instead reveals a newly generated GABAergic neuronal subtype capable of releasing GABA, remains to be determined. Both lines of DCX-CreER mice have already led to novel observations. In adult the dentate gyrus, Xiong and colleagues showed that in the inner molecular layer, the spine density of newly generated dentate granule cells increases first and then deceases one month after tamoxifen administration [6], which is in contrast to a previous finding that the spine density in the outer molecular layer continues to increase within the same time window [15]. In the adult subventricular zone, Gallo and colleagues showed that DCX-expressing neuroblasts exhibit surprising lineage plasticity and can be redirected from neuronal to glial fates, generating new oligodendrocytes in the corpus callosum after demyelination [11].

What information can we expect to gather from these DCX-CreER mice in the future? Certainly, these mice will be extremely useful for the specific and inducible genetic manipulation of newborn neurons in the adult brain. This transgenic approach creates opportunities to explore how genes of interest, such as susceptibility genes for mental disorders and neural degeneration, may regulate specific aspects of development of adult-born neurons in the intact brain. A fundamental question in adult neurogenesis concerns the function of newborn neurons in learned and affective behaviors. Due to the undesired expression of CreER in a small 
number of cells in other brain regions, including the hypothalamus around the third ventricle and the amygdala, these mice are not ideally suited to drive toxin expression to specifically knockout adult hippocampal neurogenesis during behavior analysis. However, they could potentially be used to express channelrhodopsin or halorhodopsin in a cohort of newborn dentate granule cells, which then can be selectively activated or silenced by light during behavioral testing.

The field of adult neurogenesis has gradually transitioned from the early phase of documenting basic phenomena and modulation to the level of mechanistic and functional analysis. As in any other field, the research on adult neurogenesis has been driven by both scientific and technical advances. Any effort towards generating new strategies and tools for the investigation of adult neurogenesis, such as the one by Xiong and colleagues [6], are surely welcomed by the field.

\section{Acknowledgment}

We thank Bin Wei for preparing the illustration in Figure 1, MM Song and K Christian for comments.

\section{References}

1 Ma DK, Bonaguidi MA, Ming GL, Song H. Adult neural stem cells in the mammalian central nervous system. Cell Res 2009; 19:672-682.

2 Zhao C, Deng W, Gage FH. Mechanisms and functional implications of adult neurogenesis. Cell 2008; 132:645660.

3 Kriegstein A, Alvarez-Buylla A. The glial nature of embryonic and adult neural stem cells. Annu Rev Neurosci 2009; 32:149-184.

4 Duan X, Kang E, Liu CY, Ming GL, Song H. Development of neural stem cell in the adult brain. Curr Opin Neurobiol 2008; 18:108-115.

5 Ming GL, Song H. Adult neurogenesis in the mammalian central nervous system. Annu Rev Neurosci 2005; 28:223250.

6 Cheng X, Li Y, Huang Y, Feng X, Feng G, Xiong ZQ. Pulse labeling and longterm tracing of newborn neurons in the adult subgranular zone. Cell Res 2011; 21:338-349.

7 Ge S, Goh EL, Sailor KA, Kitabatake Y, Ming GL, Song H. GABA regulates synaptic integration of newly generated neurons in the adult brain. Nature 2006; 439:589-593.

8 Mira H, Andreu Z, Suh H, et al. Signaling through BMPR-IA regulates quiescence and long-term activity of neural stem cells in the adult hippocampus.
Cell Stem Cell 2010; 7:78-89.

9 Brown JP, Couillard-Després S, Cooper-Kuhn CM, Winkler J, Aigner L, Kuhn HG. Transient expression of doublecortin during adult neurogenesis. $J$ Comp Neurol 2003; 467:1-10.

10 Couillard-Despres S, Winner B, Karl C, et al. Targeted transgene expression in neuronal precursors: watching young neurons in the old brain. Eur J Neurosci 2006; 24:1535-1545.

11 Jablonska B, Aguirre A, Raymond M, et al. Chordin-induced lineage plasticity of adult SVZ neuroblasts after demyelination. Nat Neurosci 2010; 13:541-550.

12 Schmidt-Hieber C, Jonas P, Bischofberger J. Enhanced synaptic plasticity in newly generated granule cells of the adult hippocampus. Nature 2004; 429:184-187.

13 Kempermann G, Gast D, Kronenberg G, Yamaguchi M, Gage FH. Early determination and long-term persistence of adult-generated new neurons in the hippocampus of mice. Development 2003; 130:391-399.

14 Duan X, Chang JH, Ge S, et al. Disrupted-In-Schizophrenia 1 regulates integration of newly generated neurons in the adult brain. Cell 2007; 130:1146-1158.

15 Zhao C, Teng EM, Summers RG Jr, Ming GL, Gage FH. Distinct morphological stages of dentate granule neuron maturation in the adult mouse hippocampus. J Neurosci 2006; 26:3-11. 